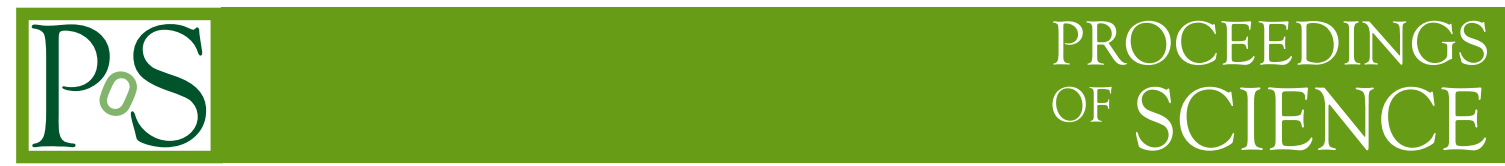

\title{
Latest results of the LHCf experiment at LHC
}

\author{
Alessia Tricomi* ${ }^{* \dagger}$ \\ University of Catania and INFN Sezione di Catania, Catania, Italy \\ E-mail: alessia.tricomiect.infn.it
}

In 2016 the LHCf experiment has fulfilled its original goal of measuring the spectra of the neutral particles produced in the very forward direction at LHC at the highest energy ever available. The main purpose of these measurements is indeed to provide the Cosmic Ray and High Energy Physics communities with a missing unique set of information for the improvement of the hadronic interaction models used to simulate air showers development produced in the interaction of primary High Energy Cosmic Rays (HECR) with the Earth atmosphere. The last data sets collected by the LHCf experiment have been obtained during $\mathrm{p}+\mathrm{p}$ collisions, at an energy of $13 \mathrm{TeV}$ in the $\mathrm{CM}$ frame, and $\mathrm{p}+\mathrm{Pb}$ collision, at an energy of the colliding nucleon pair of $5.2 \mathrm{TeV}$ and 8.1 TeV in the CM frame. A review of the main results of LHCf and of the recent and on-going activities are presented.

The European Physical Society Conference on High Energy Physics

5-12 July, 2017

Venice

* Speaker.

On behalf of the LHCf Collaboration. 


\section{Introduction}

The origin and properties of Ultra High Energy Cosmic Rays is a long standing question in astroparticle Physics. Dedicated extensive air shower experiments are in place since many years and have strongly contributed to our understanding of High and Ultra High Energy Cosmic (UHECR) Ray Physics. Recently, in particular, the Pierre Auger Collaboration [1] and the Telescope Array Collaboration [2], thanks to the excellent performance of their hybrid detector arrays, are providing us new exciting observations of UHECRs. Although these recent results have brought a deeper insight in primary cosmic ray properties, still they are largely affected by the poor knowledge of the nuclear interactions in the earth's atmosphere. In the analyses of observed data, comparison with Monte Carlo simulations of air showers is unavoidable, and the results are sensitive to the choice of hadronic interaction model used in the simulation [4]. A calibration of the energy scale in the $10^{15} \div 10^{17} \mathrm{eV}$ energy range accessible to LHC provides crucial input for a better interpretation of primary cosmic ray properties, in the region between the "knee" and the GZK cut-off. Thanks to the LHC Run1 data, some interaction models have been updated, and the dispersion found in the predictions of air shower observables decreased. However there is still significant difference between the model predictions especially in the forward region and even some analyses conclude non-realistic interpretations [3].

The Large Hadron Collider forward (LHCf) experiment was designed with the aim to provide a calibration of the hadronic interaction models in the whole energy range spanned by LHC by measuring the neutral forward particle produced in p-p as well as in p-Ion collisions. More tunings of the models using the forward particle production are necessary. In fact, the forward particles carry a large fraction of the collision energy and are responsible to determine the shape of air showers [5].

The LHCf experiment has indeed taken data in p-p collisions at $\sqrt{s}=0.9,2.76,7$ and 13 $\mathrm{TeV}$ and in $\mathrm{p}-\mathrm{Pb}$ collisions at $\sqrt{s}=5.02 \mathrm{TeV}$ thus collecting a whole set of data of unvaluable importance for the tuning of the models in such a difficult region.

\section{The LHCf experiment}

The LHCf experiment is composed by two independent position sensitive electromagnetic calorimeters, located on both side of the ATLAS experiment, $140 \mathrm{~m}$ away from the LHC-IP1 interaction point, inside the zero-degree neutral absorber (Target Neutral Absorber, TAN). Charged particles from the IP are swept away by the inner beam separation dipole before reaching the TAN, so that only photons mainly from $\pi^{0}$ decays, neutrons and neutral kaons reach the LHCf calorimeters.

Each calorimeter (ARM1 and ARM2) has a double tower structure, with the smaller tower located at zero degree collision angle, approximately covering the region with pseudo-rapidity $\eta>10$ and the larger one, approximately covering the region with $8.4<\eta<10$. Four X-Y layers of position sensitive detectors (scintillating fibers in ARM1, silicon micro-strip detectors in ARM2) provide measurements of the transverse profile of the showers. The two tower structure allows to reconstruct the $\pi^{0}$ decaying in two $\gamma_{\mathrm{s}}$, hitting separately the two towers, hence providing a very precise absolute energy calibration of the detectors. In the range $\mathrm{E}>100 \mathrm{GeV}$, the LHCf 
detectors have energy and position resolutions for electromagnetic showers better than $5 \%$ and $200 \mu \mathrm{m}$, respectively. A detailed description of the LHCf experimental set-up and of the expected physics performances can be found in Ref. [6].

\section{Results at $\sqrt{s}=13 \mathrm{TeV}$ pp collisions}

The LHCf experiment has collected data during the proton-proton collision periods at $\sqrt{s}=0.9$ $\mathrm{TeV}$ (2009 and 2010), 2.76 TeV (2013), $7 \mathrm{TeV}$ (2010) and $13 \mathrm{TeV}$ (2015).

Photon spectra at pseudorapidity $\eta$ larger than 8.8 were published for the proton-proton collision data taken at $\sqrt{s}=0.9 \mathrm{TeV}$ [7] and $7 \mathrm{TeV}$ [8]. The results were compared with the predictions by several hadronic interaction models, and it was found none of the models can perfectly match with the experimental results.

The inclusive energy spectra of photons produced in p-p collisions at $13 \mathrm{TeV}$ for two pseudorapidity ranges together with the predictions of DPMJET 3.06 [9], EPOS-LHC [10], PYTHIA 8.212 [11], QGSJETII-04 [12] and SIBYLL 2.3 [13] hadronic interaction models are shown in Fig. 1.
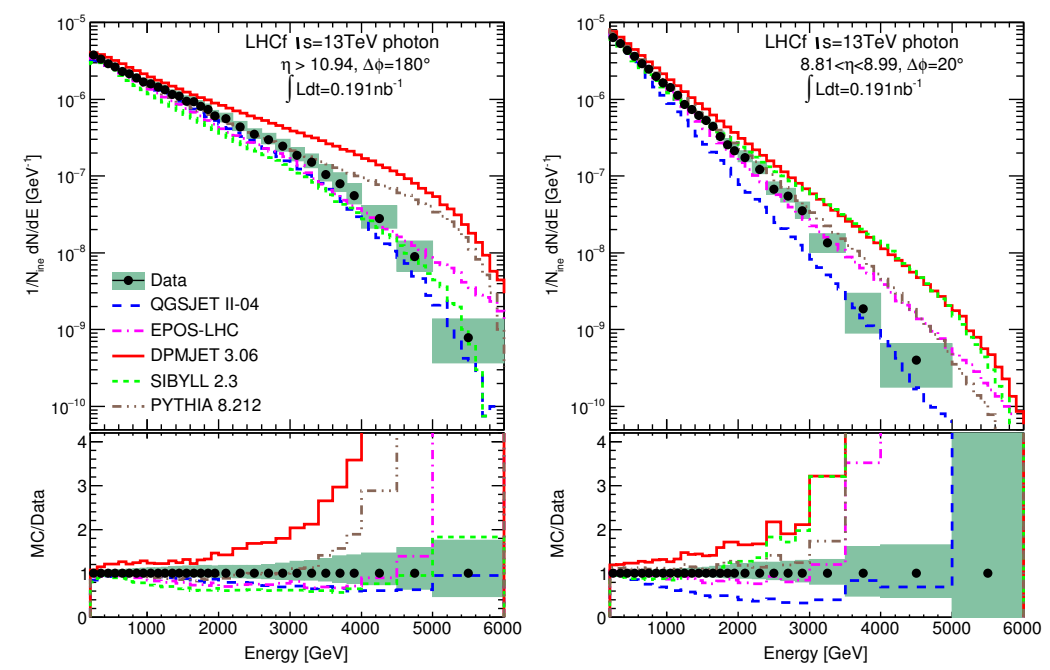

Figure 1: Inclusive photon energy spectra for pseudorapidity region $\eta>10.94$ (left) and $8.81<\eta<8.99$ (right). Data are represented by black points while MC prediction from several models are represented by coloured histograms. Green shaded area represents statistical+systematic errors of data. Spectra are normalized to the total number of inelastic collisions. Bottom panels show the ratio of MC predictions to data.

The results at $13 \mathrm{TeV}$ confirms what has been already reported at lower energies. The LHCf data lie between MC predictions but there is not an unique model with a good agreement in the whole energy range and in both rapidity regions. In the pseudorapidity range $\eta>10.94$, QGSJET and EPOS presents a good overall agreement with experimental data; SIBYLL predicts a lower yield of photons, even if it features a shape similar to data; PYTHIA spectrum agrees with data until $3.5 \mathrm{TeV}$ but become harder at higher energies; DPMJET is generally harder than data. In the pseudorapidity range $8.81<\eta<8.99$, EPOS and PYTHIA spectra agree with data until 3 
$\mathrm{TeV}$, while they become harder at higher energies; SIBYLL has a good agreement until $2 \mathrm{TeV}$, then also it becomes harder than data; QGSJET presents a lower yield of photons, while DPMJET generally predict an harder spectrum than experimental data. A deeper study of the discrepancy has brought to the conclusion that the main differences between data and models were attributable to a less-than-complete understanding of the soft hadronic interactions implemented in the models as diffractive processes $[14,15]$.

Production cross sections of neutrons were also published using the $7 \mathrm{TeV}$ proton-proton collision data [16]. The result covering 0 degree production angle showed a characteristic peak at around $2.5 \mathrm{TeV}$, and such high-energy excess was only qualitatively explained by the QGSJET II03 model. All the other models studied significantly under-predict the LHCf result. At the off-0 degree only the DPMJET model [9] well explained the LHCf results. The LHCf results showed that the ratio of energies carried by the neutrons to the photons is largest in the experimental data than any model predictions.

Results at $13 \mathrm{TeV}$ have not yet been published, however the preliminary spectra obtained for the forward neutron production using the ARM2 detectors, shown in Fig. 2, seems to confirm the same trend observed at $7 \mathrm{TeV}$, in particular, the peak at 0 -degree for energy $\simeq 5.5 \mathrm{TeV}$ in the data which is reproduced only qualitatelively by the QGSJET II-03 model while all the other models studied significantly under-predict the LHCf result. At the off- 0 degree the agreement between data and models is also poor.
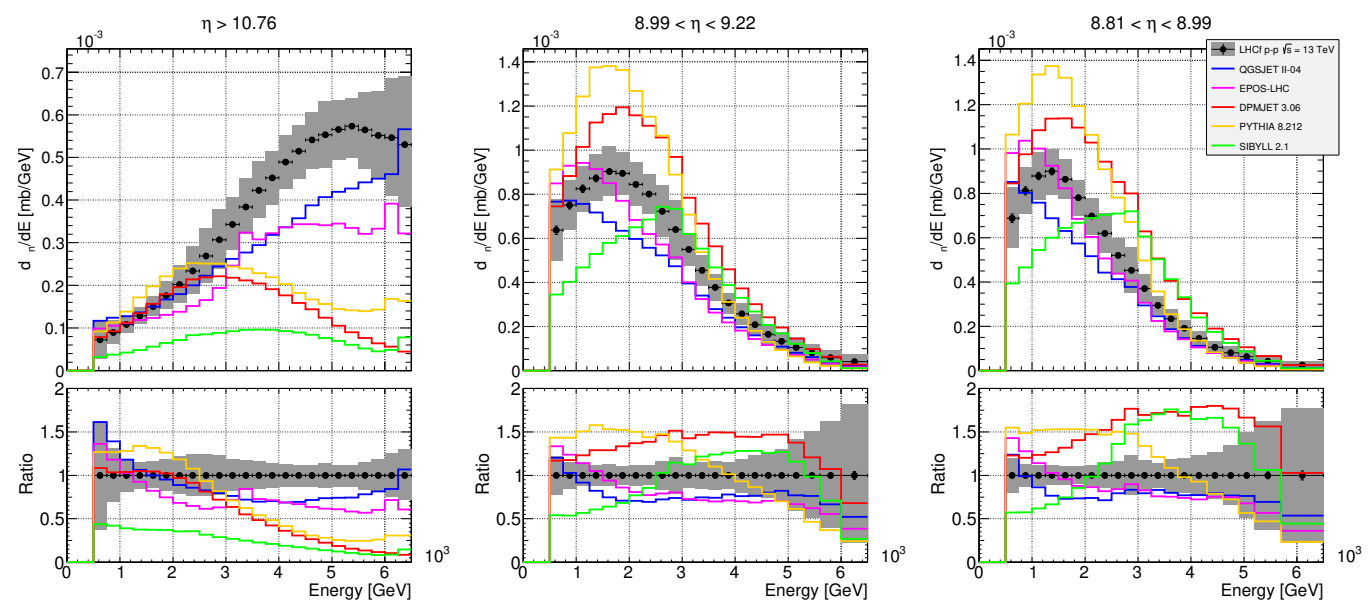

Figure 2: Inclusive neutron energy spectra of ARM2 detector for pseudorapidity region $\eta>10.76$ (left), $8.99<\eta<9.22$ (center) and $8.81<\eta<8.99$ (right). Data are represented by black points while MC prediction from several models are represented by coloured histograms. Grey shaded area represents statistical+systematic errors of data. Spectra are normalized to the total number of inelastic collisions. Bottom panels show the ratio of MC predictions to data.

This measurement is particularly important since it is expected to provide an important hint for the problem of cosmic-ray muon excess reported by both Auger [17] and TA [18]. This excess could be indeed interpreted in terms of an underestimation of the baryon production in the hadronic interaction models used to derive the CR spectra [19]. 


\section{Summary and Plans}

The LHCf experiment has successful taken data at various collision condition at LHC thus providing a unique set of measurements in the before-unexplored forward region to constraint the particle production relevant for the high energy Cosmic Ray physics. The so far published photon, neutron and $\pi^{0}$ results are compared with the interaction models providing unvaluable input for the models tuning. The discrepancy found between data and models may related to the diffractive/nondiffractive components and may benefit of combined analysis between LHCf and ATLAS which will be the aim of future work. In addition, the comparisons between the data at different collision energies reveal the scaling or scaling break of the partile spectra. The LHCf results indicate the scaling of photons and $\pi^{0}$ spectra at $\sqrt{s}=2.76,7$ and $13 \mathrm{TeV}$, while the ISR, PHENIX and LHCf indicate a scaling break of neutron spectra between $200 \mathrm{GeV}$ and $13 \mathrm{TeV}$. A new experiment, RHICf, has hence been prepared to test the scaling (or break) at $\sqrt{s}=510 \mathrm{GeV}$ at RHIC. The experiment uses one of the LHCf detector, ARM1, and has taken data at the end of June. Results from RHICf will come in the near future and will bring further important information from Cosmic Ray physics.

\section{References}

[1] F. Fenu [Pierre Auger Collaboration], Proc. of 35th Int. Cosmic Ray Conf., Bexo, Busan, Korea (2017), C17-07-12, p. 9-16.

[2] R. U. Abbasi et al. [Telescope Array Collaboration], Astropart. Phys. 80 (2016) 131

[3] Pierre Auger Collaboration, Phys. Rev. D 90, 122005 (2014).

[4] K.-H. Kampert and M. Unger, Astropart. Phys. 35 (2012) 660.

[5] K. Akiba et al., J. Phys. G: Nucl. Part. Phys. 43 (2016) 110201.

[6] O. Adriani et al., JINST 3 (2008) S08006.

[7] O. Adriani et al., Phys. Lett. B715 (2012) 298.

[8] O. Adriani et al., Phys. Lett. B703 (2011) 128.

[9] F.W. Bopp et al., Phys. Rev. C77 (2008) 014904.

[10] K. Werner et al., Nucl.Phys.Proc.Suppl. 175-176 (2008)

[11] T. Sjöstand, et al., Comput. Phys. Comm. 178 (2008) 852.

[12] S. Ostapchenko, Phys. Rev. D83 (2011) 014108.

[13] E.-J. Ahn et al., Phys. Rev. D80 (2009) 094003.

[14] The ATLAS and the LHCf Collaborations, ATL-PHYS-PUB-2015-038.

[15] Q.D. Zhou, Y. Itow, H. Menjo and T. Sako, Eur. Phys. J. C 77 (2017) 212.

[16] O. Adriani et al., Phys. Lett. B 750 (2015) 360.

[17] P. Abreu et al. [The Pierre Auger Collaboration], Proc. of 32th Int. Cosmic Ray Conf., Beijing (2011), [arXiv:1107.4804 [astro-ph.HE]].

[18] T. Abu-Zayyad, et al. [TA Collaboration], Astrophys. J. 768 (2013) L1

[19] T. Pierog and K. Warner, Phys. Rev. Lett. 101 (2008) 171101. 